Mark Leininger

April 1982

Abstract

Hand calculations made so far on the Central Calorimeter Cradle Assembly (Fig. 1) are compared with stress and displacement solutions obtained using ANSYS.

\title{
Introduction
}

The Central Calorimeter Cradle Assembly as shown in Fig. 1 has a weight of approximately 200 tons. It is made of 12 modules (Fig. 2) bolted together near their outer radius supported by a steel cradle (Fig. 3). The purpose here is to analyze this structure in two dimensions, looking in particular at:

I. The stresses in the cradle at $\theta=165^{\circ}$ where it is thin (Fig. 4).

II. The shear stresses in the keys between modules, especially at $\theta=120^{\circ}$ (Fig. 4).

III. The deflection at the top module (Fig. 4). 


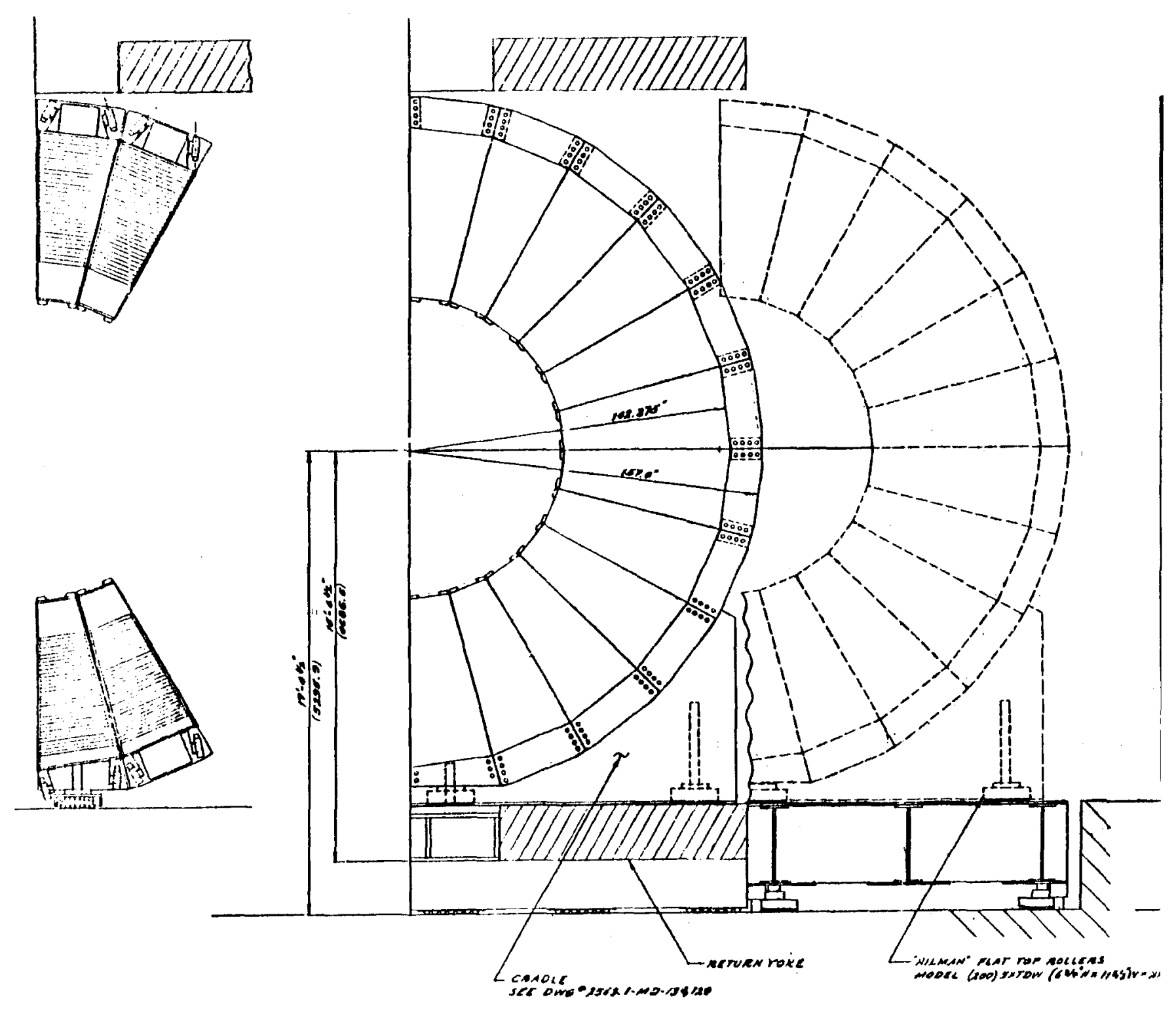

Fig. I 


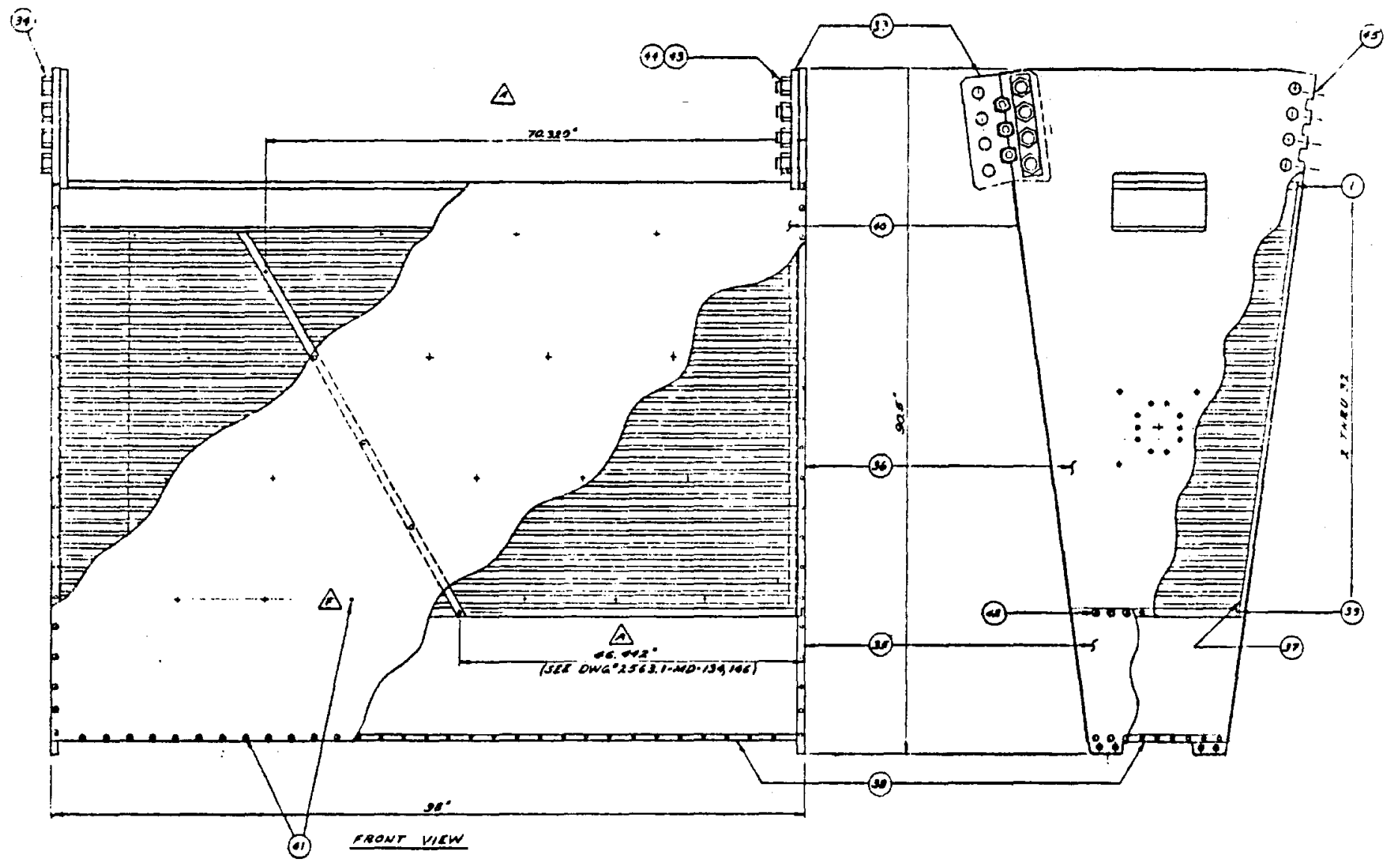

Fig. 2 


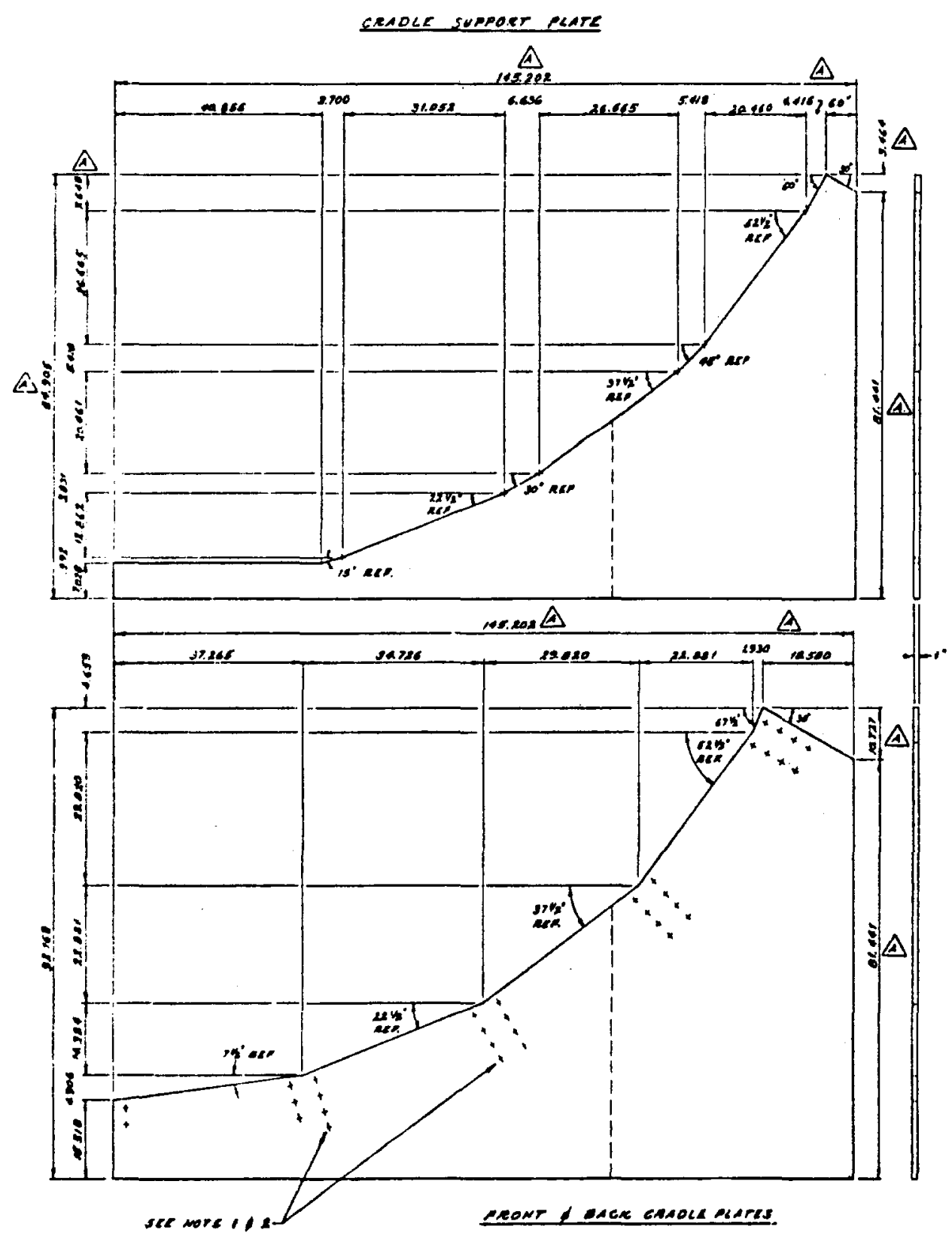

Fig. 3 

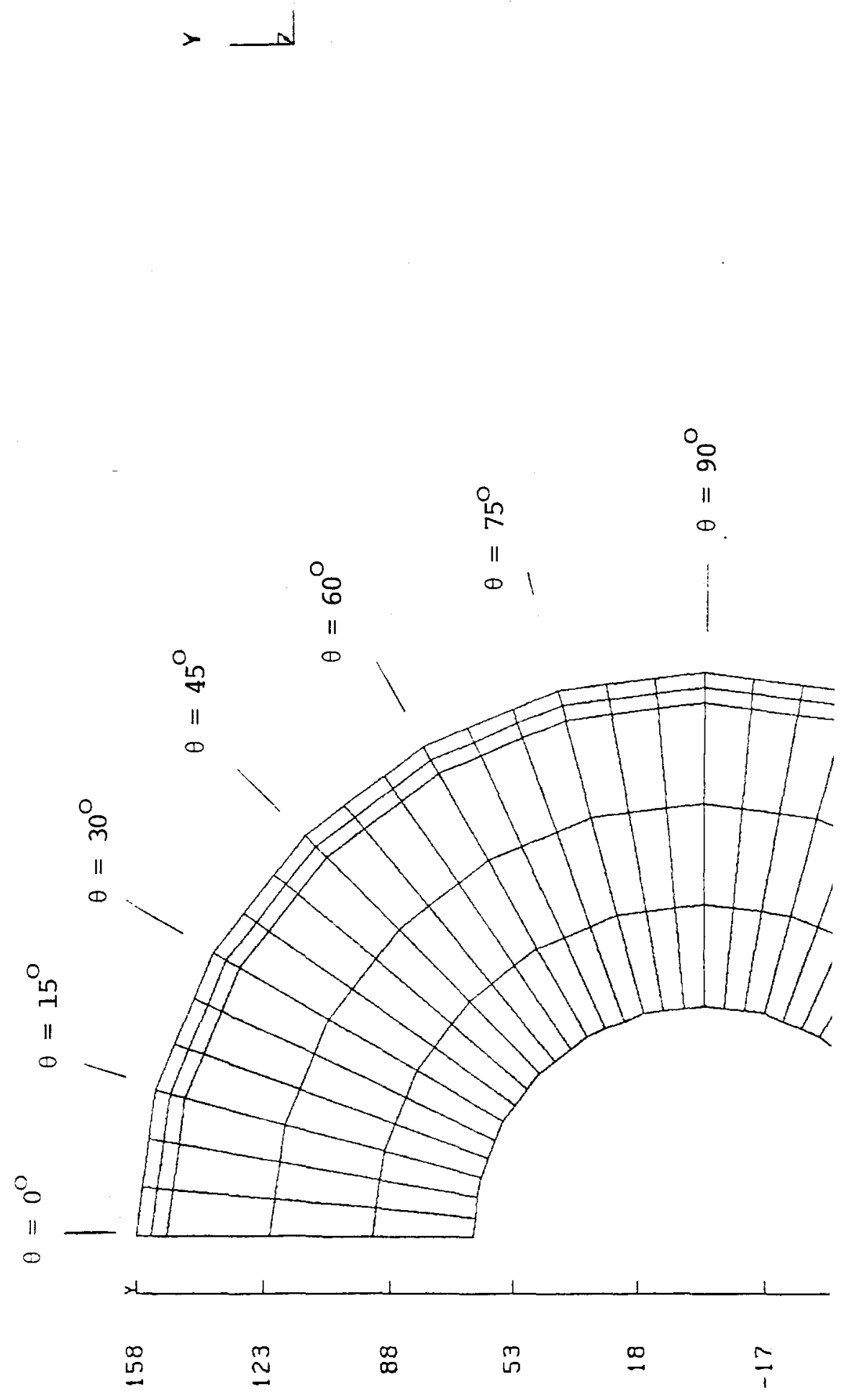


\section{Stresses in Cradle}

This problem was solved by Professor Lovell at the University of Wisconsin using a model in which module 1 does not interact with any other modules (Fig. 5).
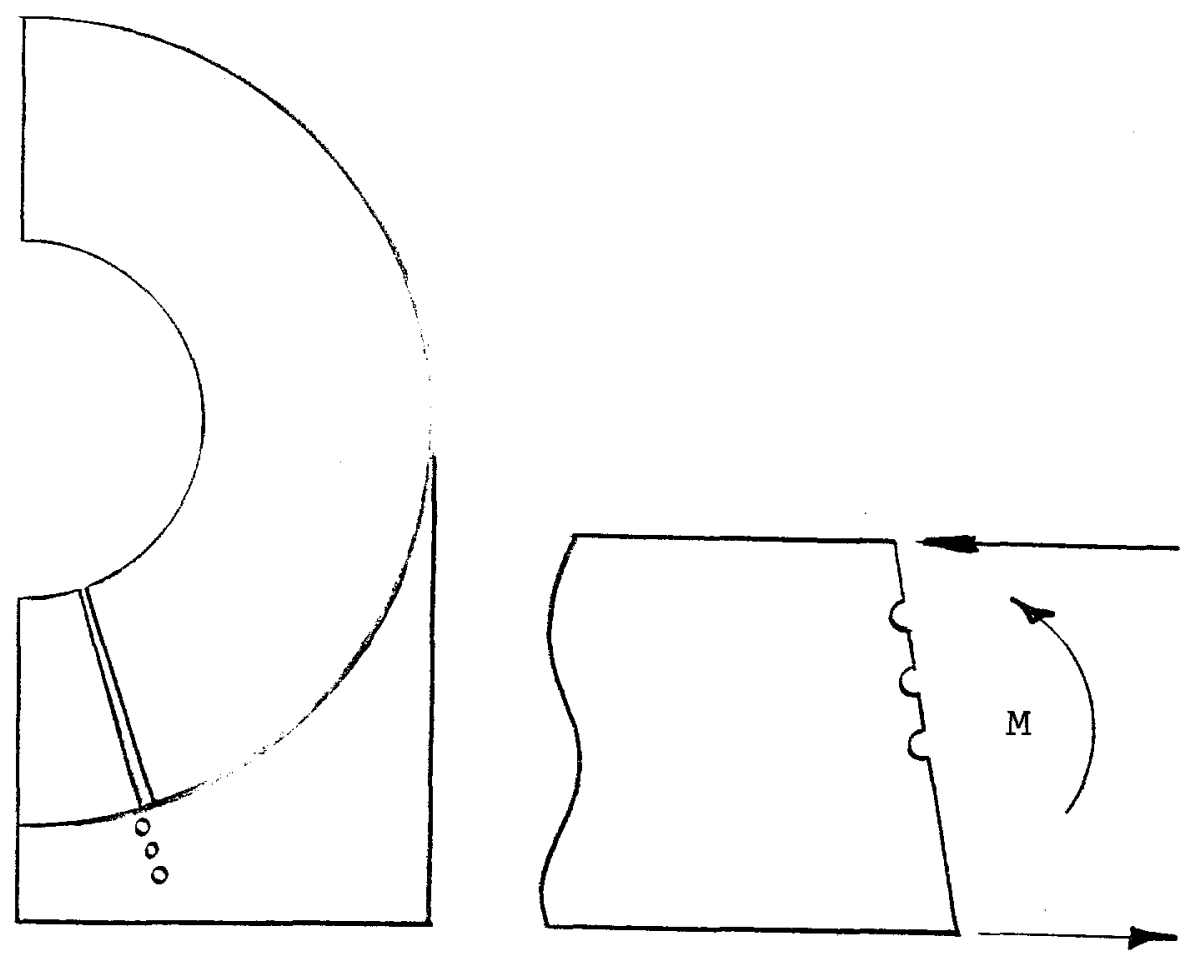

Maximum compressive stress

Maximum tensile

Fig. 5

The mament to he computes at the bolt hole line is

$$
\begin{aligned}
& M=1.48 \mathrm{E} 6 \text { in } 16 . \text { which results in stresses of } \\
& \sigma_{x}=+15,490 \text { psi (tension } \\
& -28,760
\end{aligned}
$$


The ANSYS solution shows stresses much lower and of the opposite sign in this region (Fig. 6), the max stress shown being $\sigma_{x}=-2905$ psi. To resolve this difference, it is necessary to take into consideration the effect of the other modules on module 1 (Fig. 7). The numbers shown are extracted from the ANSYS solution.

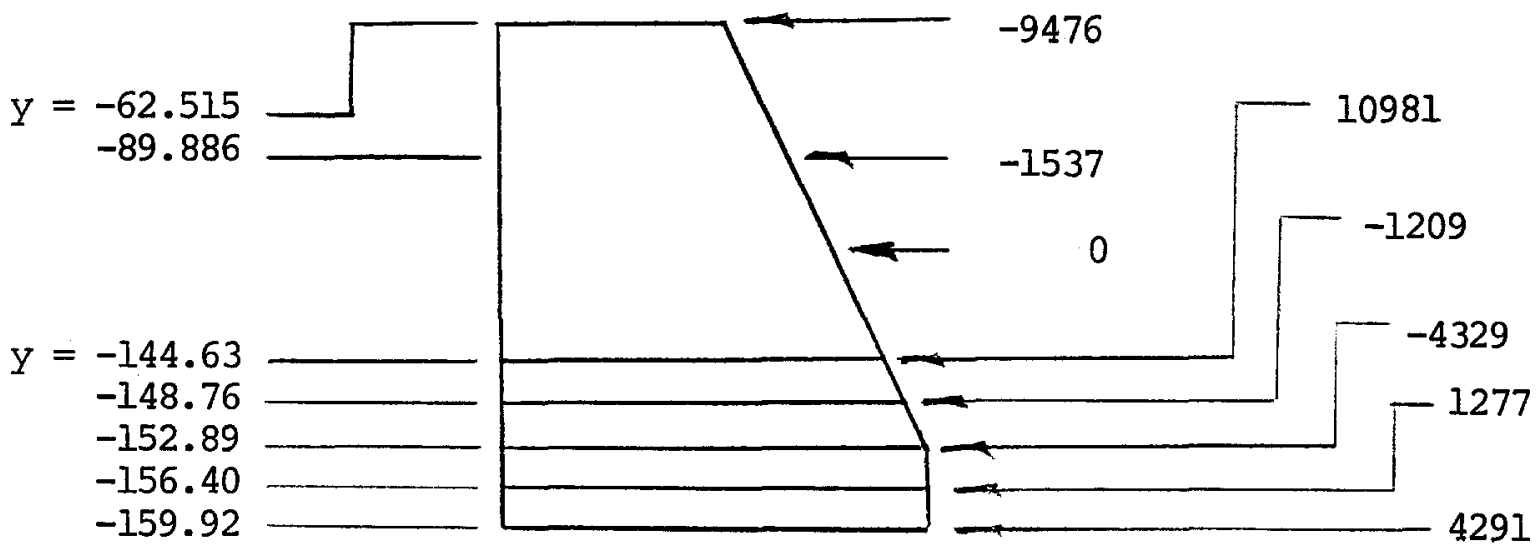

Fig. 7

Reaction forces shown are forces on nodes due to adjacent elements. Neutral axis:

$$
y_{C}=-\left(\frac{159.92-144.63}{2}\right)-144.63=-152.275
$$

Moment on section shown (without STIF 12 terms)

$$
\begin{aligned}
+\int \sum M= & (-10981)(152.275-144.63)+(+1209)(152.275-148.76) \\
& +(-4329)(152.89-152.275)+(+1277)(156.40-152.275) \\
& +(+4291)(159.92-152.275) \\
= & -4.43 \mathrm{E} 4 \text { in } 1 \mathrm{~b} .
\end{aligned}
$$

Contribution due to STIF 12 terms:

$$
\begin{aligned}
+\sum \sum M & =(9476)(152.275-62.215)+(1537)(152.275-89.886) \\
& =9.49 \text { E5 in } 1 \mathrm{~b} .
\end{aligned}
$$


Fig. 6

STEP $=1$ ITER $=10 \quad$ TIME $=.00$

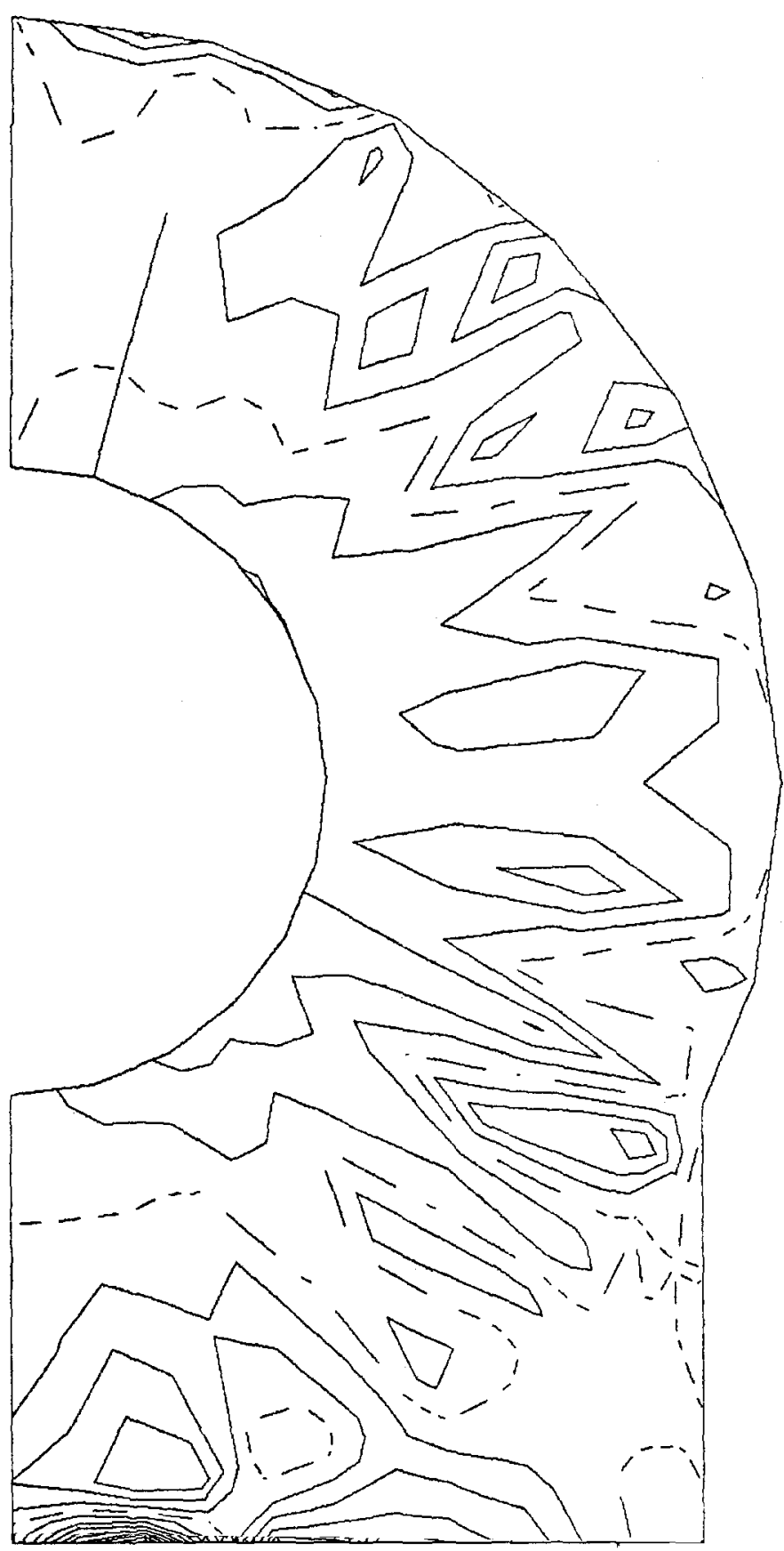


If the moment generated by the STIF 12 elements is subtracted out of the section of interest, the result is:

$$
+\int \sum \mathrm{M}=9.49 \mathrm{E} 5-4.43 \mathrm{E} 4=9.05 \mathrm{E} 5 \text { in } \mathrm{lb} \text {. } \frac{\text { compression }}{\text { on top of beam }}
$$

It is clear that the effect of the other modules on module $I$ is large, and that when the effect of those modules is subtracted from the ANSYS solution, the resulting moment is $9.05 \mathrm{E} 5 \mathrm{in} \mathrm{lb}$. compared with $1.48 \mathrm{E} 6$ in $\mathrm{lb}$. computed by Lovell. It appears that this is one case where the model used was an oversimplification of the real problem. 
II. Shear Stresses in Keys Between Modules

The shear stresses in the keys between modules are a point of concern, particularly at the location $\theta=120^{\circ}$ (Fig. 4). There was some question as to whether the cradle needed to be extended high enough to capture this module and the answers from both ANSYS and a hand solution done by cook at the University of Wisconsin indicate that it is imperative that the cradle be extended high enough to resist the shear at this location. Table I summarizes the shear force and shear stress in keys between each pair of modules. Note that shear is assumed to be taken by all three elements of the key which requires fairly close attention to the tolerances on these parts.

\section{Table I}

Shear Force (lb.)

$\underline{\theta}$

15

30

45

60

75

90

105

120

\section{Cook}

15455

27673

33904

32010

20709

0

28955

63959
ANSYS

$$
16590
$$

29752

36438

34354

22228

0.2

31119

67329
Shear Stress (psi)

Cook

3434

ANSYS

3687

6150

6612

7534

8097

7113

7634

4602

4940

0

0

6434

6915

14213 
III. Deflection at Top Module

This question is of importance because of the size of the elements inside the bore of the calorimeter. If the top module sags too much, it will rest on the coil inside. The deflection has been computed by cook for a solid curved beam constrained at $\theta=3 \pi / 4$ (measured from top), by the author (using Castiglianos Theorem), and by ANSYS with and without friction between modules. The basic scheme for the hand calculation is as follows:

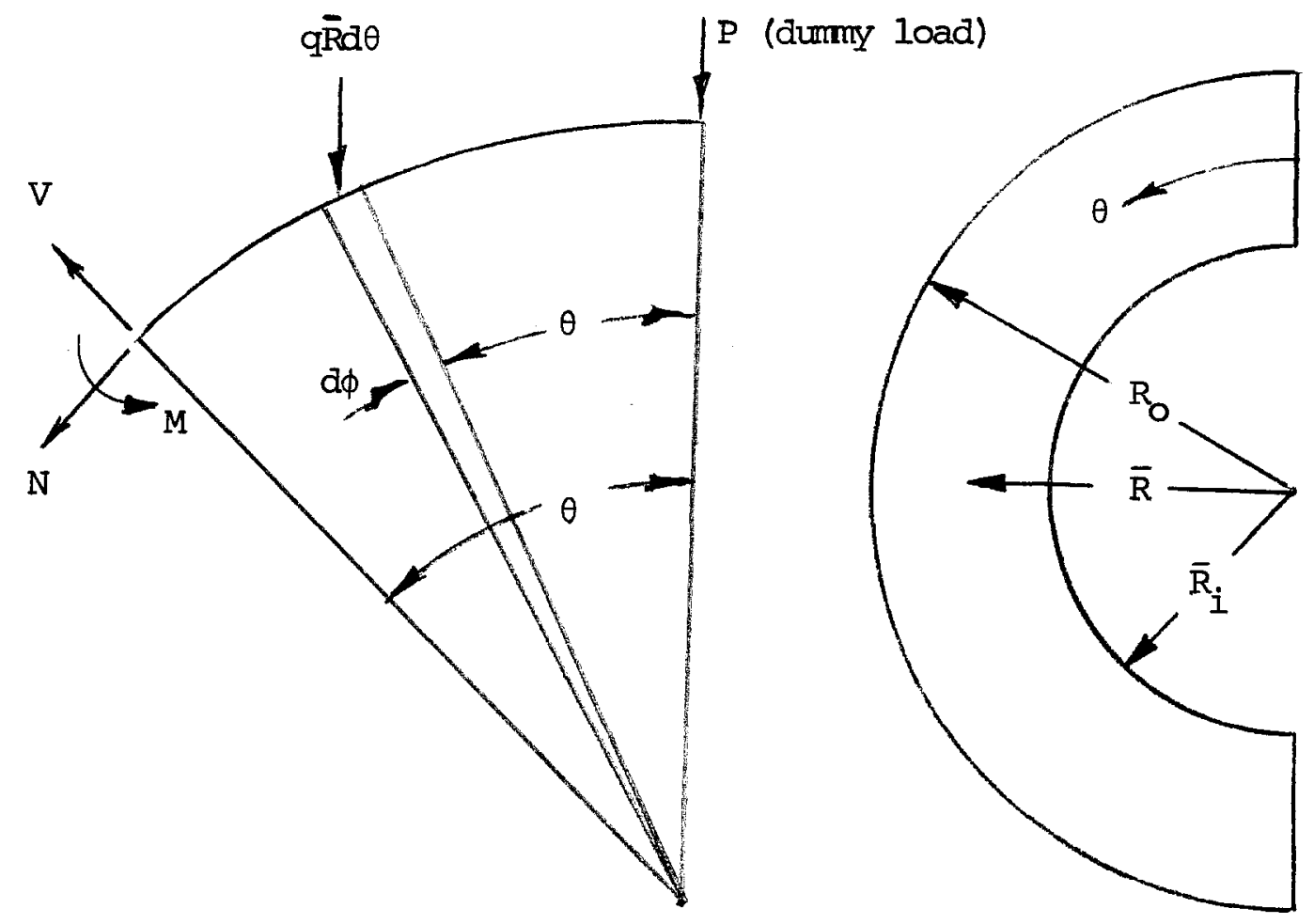

Static equilibrium gives:

$$
\begin{aligned}
& \mathrm{N}=-P \sin \theta-\mathrm{q} \overline{\mathrm{R}} \theta \sin \theta \\
& \mathrm{V}=P \cos \theta+\mathrm{q} \overline{\mathrm{R}} \theta \cos \theta \\
& \mathrm{M}=P R \sin \theta+\mathrm{q} \overline{\mathrm{R}}^{2}(\theta \sin \theta+\cos \theta-1)
\end{aligned}
$$


Vertical deflection $\Delta \mathrm{V}$ will then be

$$
\begin{aligned}
& \Delta V=\frac{\partial U}{\partial P} \text { where } U \text { is strain energy: } \\
& U=\int_{0}^{\pi} \frac{k_{V} V^{2}}{2 A G} R d \theta+\int_{0}^{\pi} \frac{k_{N} N^{2}}{2 A E} R d \theta+\int_{0}^{\pi} \frac{k_{M} M^{2}}{2 E I} R d \theta
\end{aligned}
$$

where $\mathrm{A}=$ cross sectional area

$\mathrm{G} \quad=$ shear modulus

$E \quad=$ Young's modulus

I = moment of inertia

$\mathrm{k}^{\prime} \mathrm{s}=$ correction factors due to fact this is not a continuous, homogeneous beam.

The results are shown in Table II.

Table II

Deflection of Top Module

Horizontal

Vertical

Deflection (in).

Deflection (in.)

Cook solution (hand)

$-0.150$

$-0.188$

Author solution (hand)

$-0.066$

ANSYS:

As solid beam

$-0.048$

$-0.039$

As modules, $\mathrm{MU}=0.0$

$-0.179$

$-0.142$

As modules, $\mathrm{MU}=0.2$

$-0.140$

$-0.107$ 
Table II presents horizontal and vertical deflection for the cases of Cook's hand solution, the author's hand solution for $\theta=0$ to $\pi$, ANSYS solution treating arch as solid beam of steel (same model as Cook), ANSYS solution using modules with $\mathrm{MU}=0.0$, ANSYS solution using modules with $\mathrm{MU}=0.2$.

First it is of interest to note that the arch under consideration will deflect further vertically if constrained at $\theta=3 \pi / 4$ ( $\theta=0$ at top) than it will if constrained at $\theta=\pi$. This accounts for the difference between Cook's and the author's solution.

The other item of interest is the effect of friction in the ANSYS solutions. Added friction reduces deflections in both directions.

The conclusion to be made is that deflections of the order of $1 / 8 \mathrm{in}$. are expected.

Comments on Figs. 8-15

All of the figures 8-15 are plots of the ANSYS solution for the case of MU $=0.2$. Fig. 8 shows the displacement as a solid outline, the undeflected geometry as a dashed outline. Figs. 9 and 10 represent normal stress in the $\mathrm{x}$ and $\mathrm{y}$ directions. Note that none of the stress output is meaningful in the modules because the algorithm used to compute stresses interpolates across the gaps as if they weren't there. The stress information in the cradle is accurate because it is continuous. Fig. Il shows the shear stresses in the xy plane. Figs. 12 and 13 shows maximm and minimum stresses in the principal directions. Fig. 14 is maximm shear stress, and Fig. 15 is equivalent stress (Von Mises stress). 


\section{Conclusions}

1. The cradle must be constructed to support shear at $\theta=120^{\circ}$.

2. Stresses in the thin part of the cradle are not excessive for the assembled structure. Some consideration should be given to assembly technique to ensure minimm stresses during assembly of modules to cradle.

3. The components internal to the calorimetry modules must allow the modules at the top to sag at least $1 / 8$ in.

\section{Acknowledgments}

Thanks to Bob Wands for assistance in running ANSYS and interpreting its output, and John Grimson for help with analytic solution. 
Fig. 8

STEP $=1$ ITER $=10$ TIME $=.00$

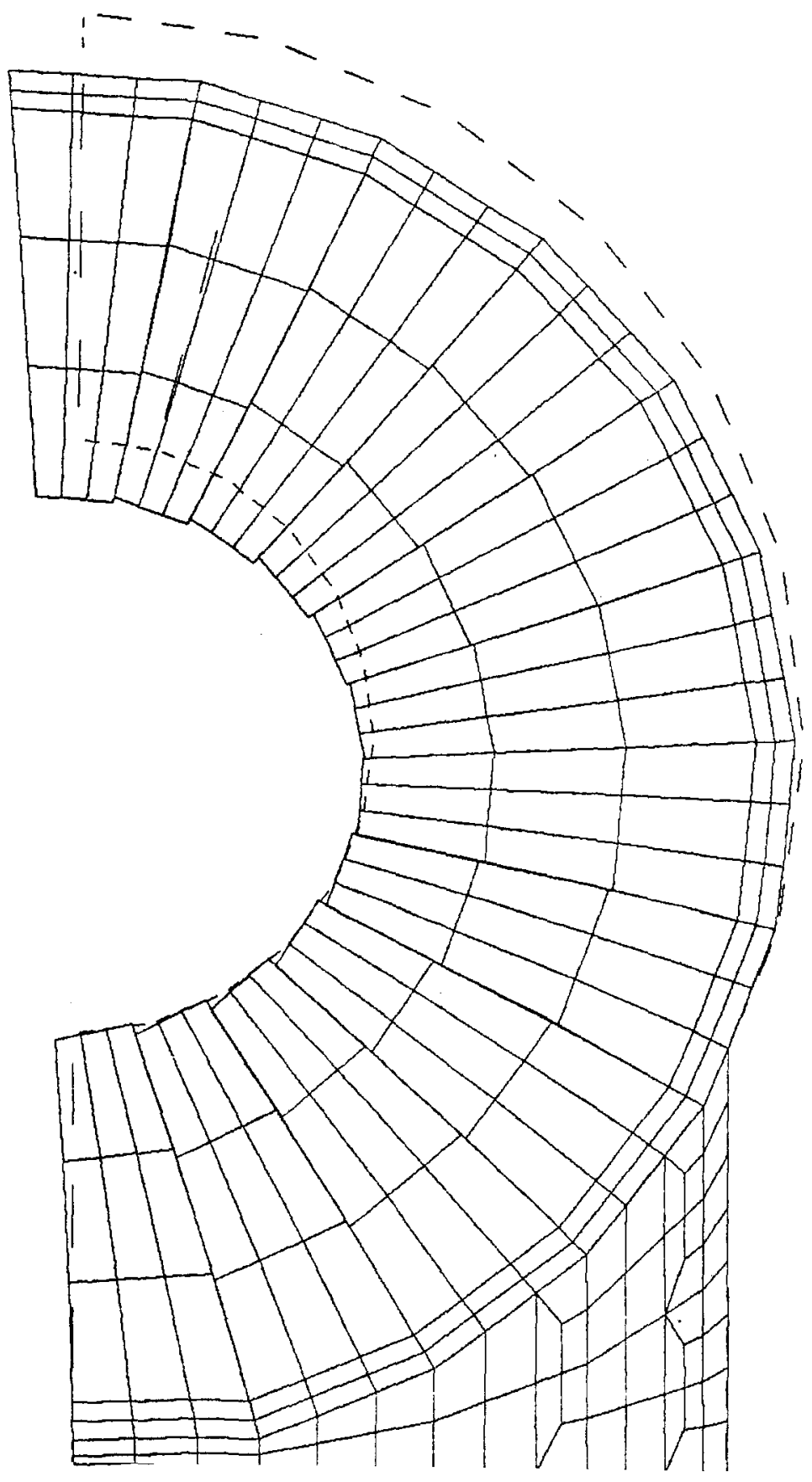


Fig. 9

STEP $=1$ ITER $=10$ TIME $=.00$

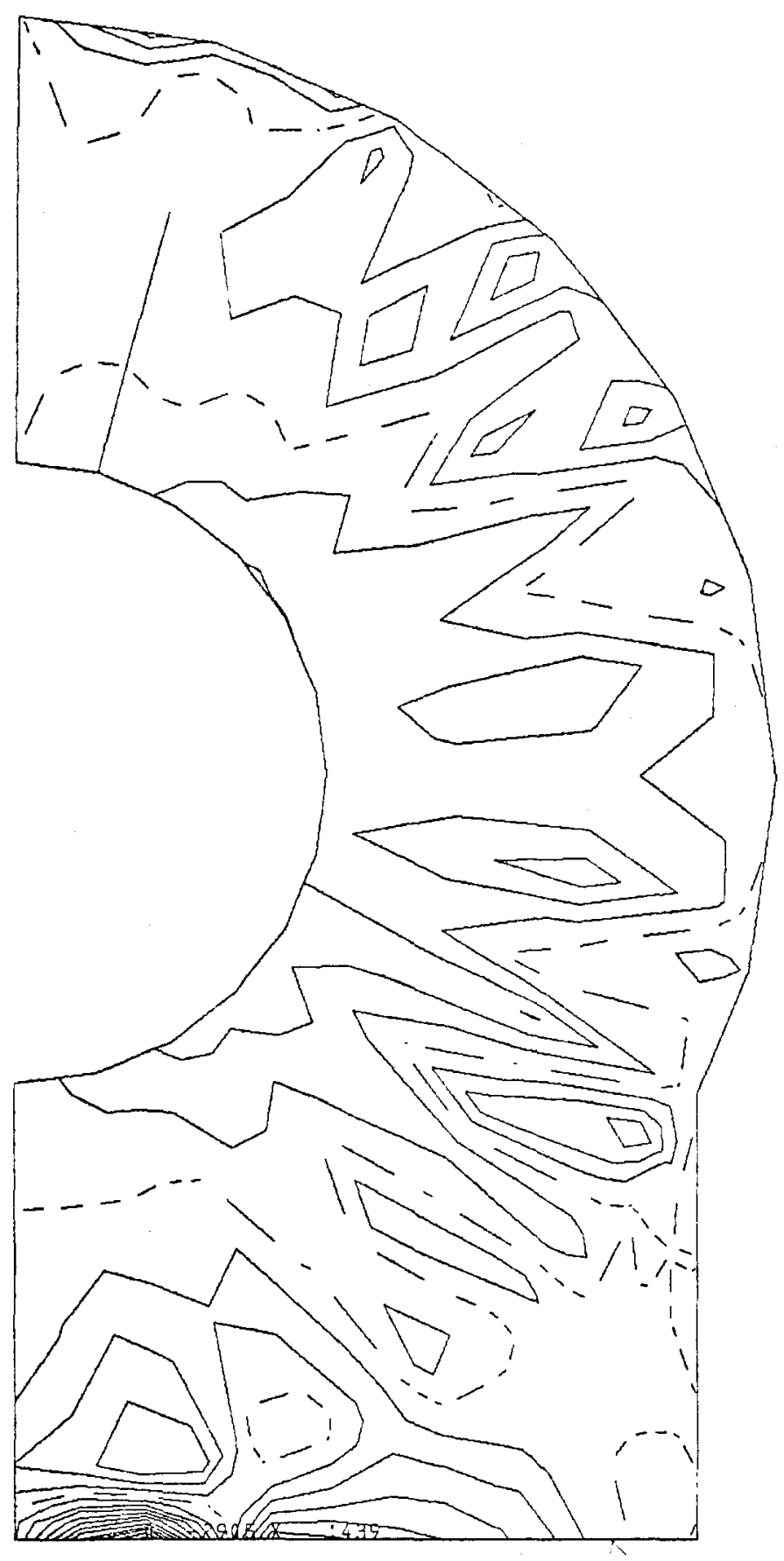


Fig. 10

$$
\text { STEP }=1 \text { ITER }=10 \text { TIME }=.00
$$

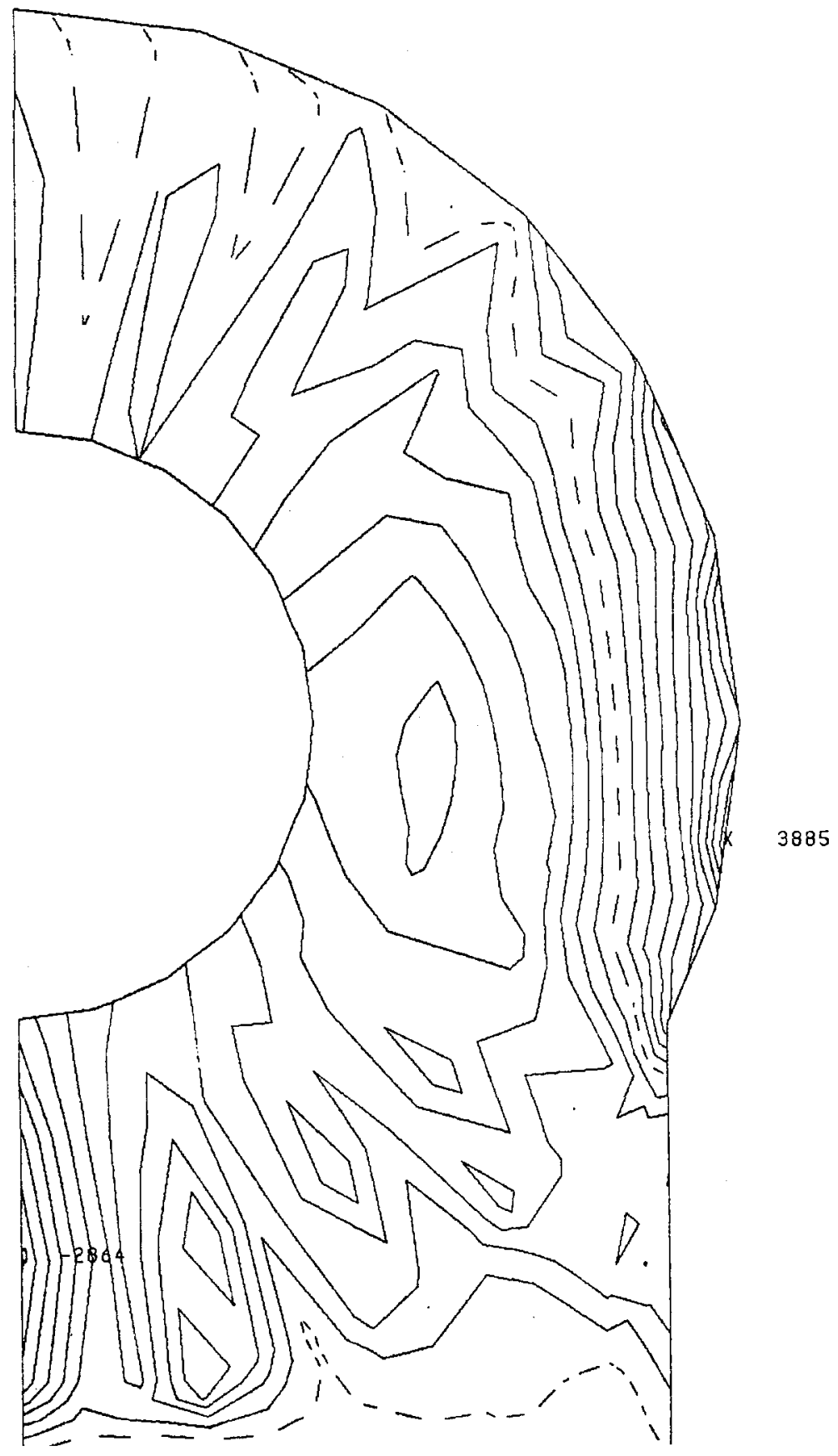


Fig. 11

STEP $=1$ ITER $=10$ TLME $=.00$

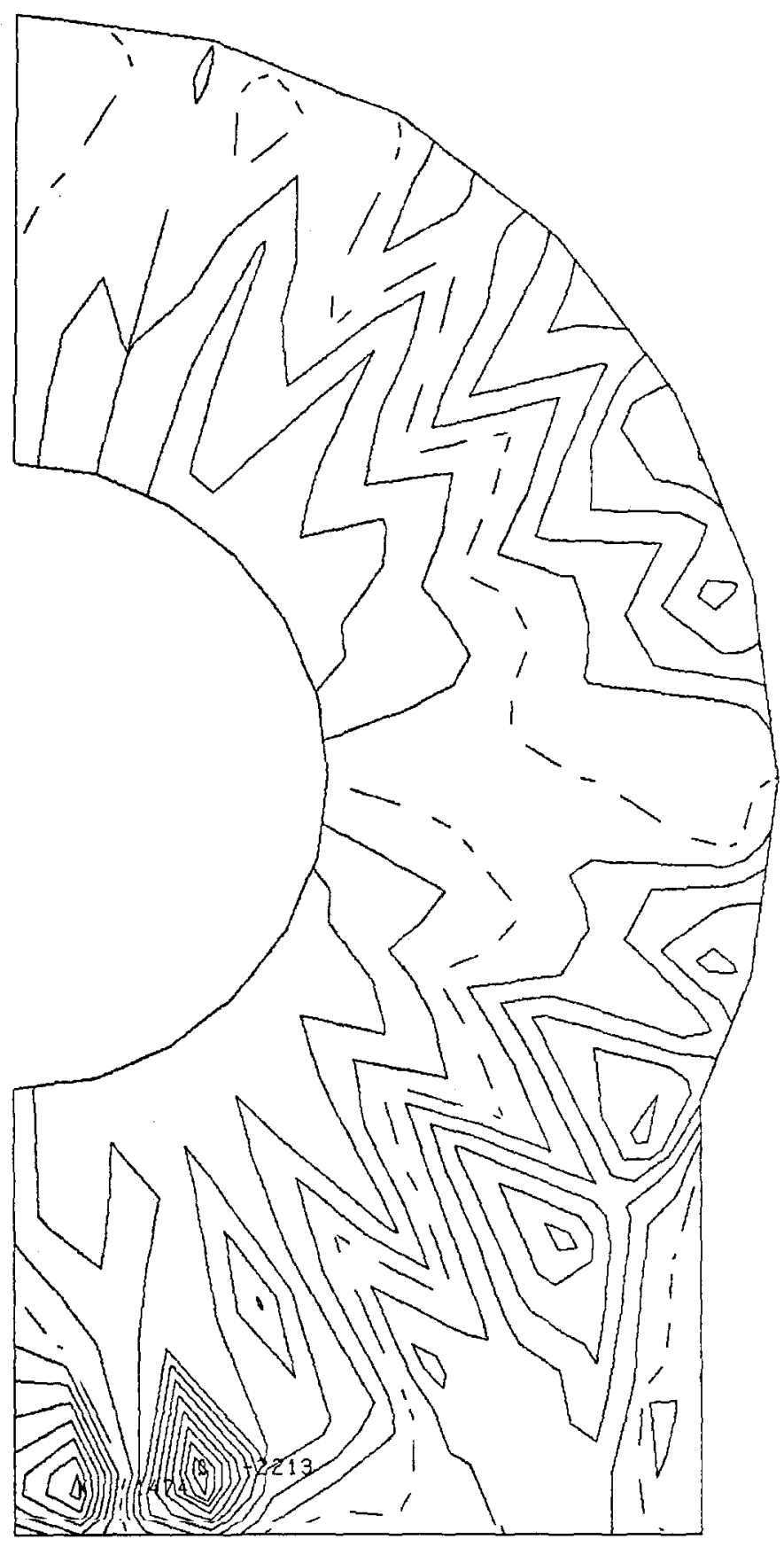




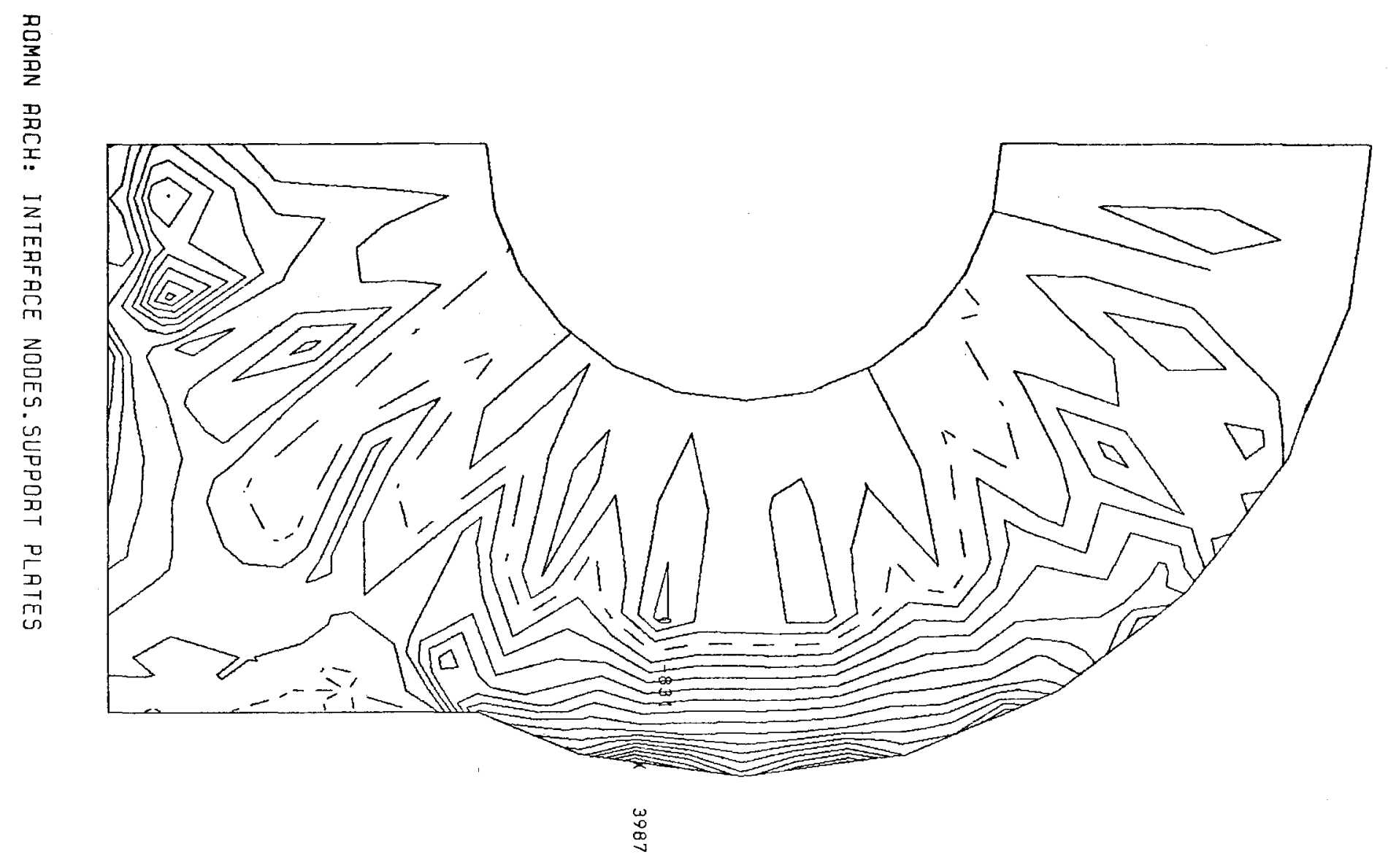


Fig. 13

STEP $=1$ ITER $=10 \quad$ TIME $=.00$

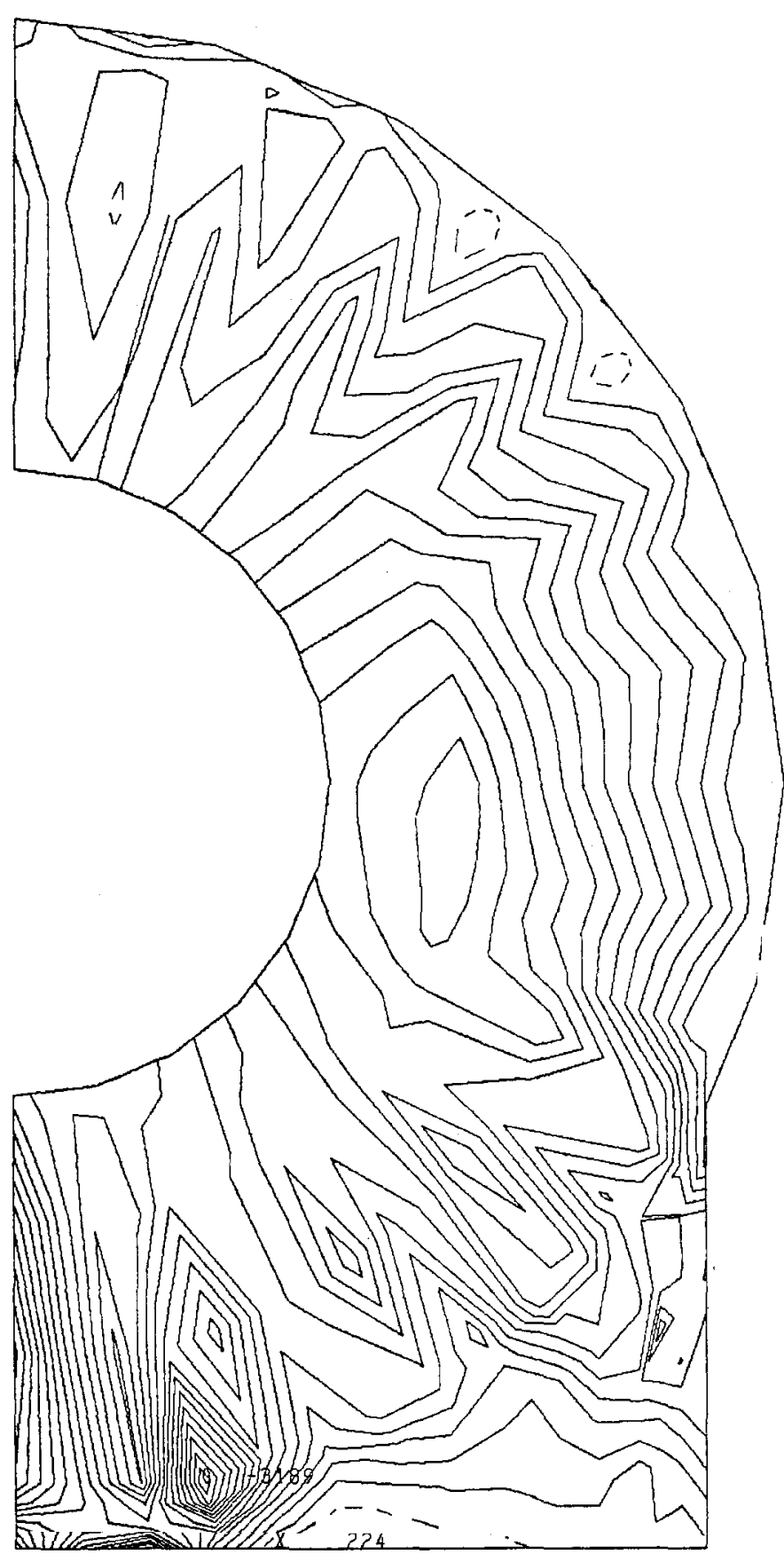


Fig. 15

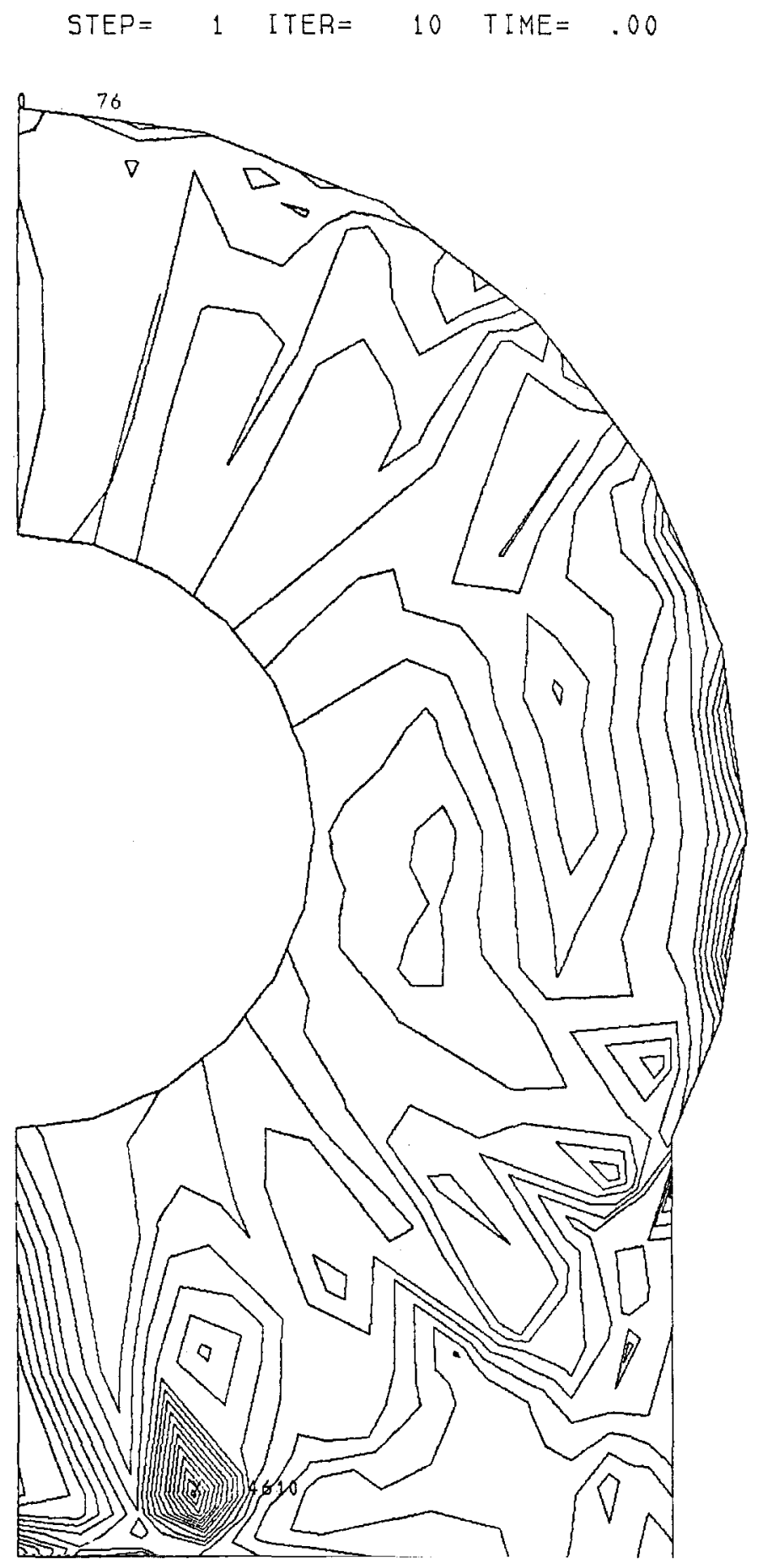

